\title{
CHARACTERIZATIONS OF DENTING POINTS
}

\author{
BOR-LUH LIN, PEI-KEE LIN ${ }^{1}$ AND S. L. TROYANSKI
}

(Communicated by William J. Davis)

\begin{abstract}
Let $x$ be a PC (point of continuity) for a bounded closed convex set $K$ of a Banach space. Then $x$ is a denting point of $K$ if and only if $x$ is an extreme point (resp. strong extreme point; weak*-extreme point) of $K$. A new definition for denting point is also given.
\end{abstract}

Throughout this paper, $K$ is a bounded closed convex set of a Banach space $X$. An element $x$ in $K$ is called a denting point of $K$ if $x \notin \overline{c o}(K \backslash B(x, \varepsilon)$ ) for all $\varepsilon>0$ where $B(x, \varepsilon)=\{y: y \in X,\|y-x\|<\varepsilon\} . x$ is a PC (point of continuity) for $K$ if the identity mapping $(K$, weak $) \rightarrow(K$, norm $)$ is continuous at $x$.

Recall that $x$ is a strong extreme point of $K$ if for any sequences $\left\{y_{n}\right\}$ and $\left\{z_{n}\right\}$ in $K, \lim _{n \rightarrow \infty}\left\|\frac{1}{2}\left(y_{n}+z_{n}\right)-x\right\|=0$ implies that $\lim _{n \rightarrow \infty}\left\|y_{n}-x\right\|=0$. $x$ is a weak*-extreme point of $K$ if $x$ is an extreme point of $\tilde{K}$ where $\tilde{K}$ is the weak*closure of $K$ in $X^{* *}$. A Banach space $X$ is said to have the Kadec property (K) (resp. property $(\mathrm{G})$; midpoint locally uniform rotundity) if every point on the unit sphere of $X$ is a PC (resp. denting point; strong extreme point) for the closed unit ball of $X$ [LL, T]. It has been proved [LLT1] that a Banach space $X$ has property (G) if and only if $X$ is strictly convex and $X$ has property $(\mathrm{K})$. It is easy to see that every denting point of $K$ is a strong extreme point of $K$ and it is known [KR] that every strong extreme point of $K$ is a weak*-extreme point of $K$.

DEFINITION. An element $x$ in $K$ is called a very strong extreme point of $K$ if for every sequence $\left\{x_{n}\right\}$ of $K$-valued Bochner integrable functions on $[0,1]$, the conditon

$$
\lim _{n \rightarrow \infty}\left\|\int_{0}^{1} x_{n}(t) d t-x\right\|=0 \text { implies } \lim _{n \rightarrow \infty} \int_{0}^{1}\left\|x_{n}(t)-x\right\| d t=0 .
$$

It is well known that if $x$ is a denting point of $K$ then $x$ is a PC for $K$ and $x$ is an extreme point of $K$. In [LLT2] (or see [R]), we show that the converse is true. In fact, we have the following results.

THEOREM. Let $x$ be an element in a bounded closed convex set $K$ of a Banach space. Then the following are equivalent:

(i) $x$ is a denting point of $K$.

(ii) $x$ is a very strong extreme point of $K$.

(iii) $x$ is a $P C$ for $K$ and $x$ is an extreme point (resp. strong extreme point, weak*-extreme point) of $K$.

Received by the editors June 23, 1986 and, in revised form, November 12, 1986.

1980 Mathematics Subject Classification (1985 Revision). Primary 46B20.

Key words and phrases. Denting point, extreme point, strong extreme point.

${ }^{1}$ Research partially supported by NSF DMS-8403669. 
A subset $S$ of $K$ is an open slice if there are a nonzero $x^{*}$ in $X^{*}$ and $\varepsilon>0$ such that $S=\left\{x: x \in K, x^{*}(x)>\sup x^{*}(K)-\varepsilon\right\}$. It is well known that $x$ is a denting point of $K$ if and only if for any $\varepsilon>0$ there exists a slice $S$ of $K$ such that $x \in S$ and diameter of $S$ is less than $\varepsilon$. From the following lemma, if $x$ is a PC for $K$ and if $x$ is a weak*-extreme point of $K$ then $x$ is a denting point of $K$. The authors wish to thank Professor H. P. Rosenthal for providing the lemma. We include a proof of the lemma for the sake of completeness.

LEMMA. Let $x$ be an element in a bounded closed convex set $K$ of a Banach space. Then $x$ is a weak ${ }^{*}$-extreme point of $K$ if and only if the open slices containing $x$ form a neighborhood base for $x$ in the weak topology on $K$.

ProOF. Suppose $x$ is a weak*-extreme point of $K$. Let $V=\left\{y: y \in K, x_{i}^{*}(y)-\right.$ $\left.x_{i}^{*}(x)<\varepsilon, i=1,2, \ldots, n\right\}$ be a weak neighborhood of $x$ in $K$. For $i=1,2$, let $M_{i}=\left\{y: y \in K, x_{i}^{*}(y)-x_{i}^{*}(x) \geq \varepsilon\right\}$. Suppose $x \in \overline{\mathrm{co}}\left(M_{1} \cup M_{2}\right)$. Then there exist $y_{n} \in M_{1}, z_{n} \in M_{2}$ and $\alpha_{n} \in[0,1]$ such that $\lim _{n \rightarrow \infty}\left\|\alpha_{n} y_{n}+\left(1-\alpha_{n}\right) z_{n}-x\right\|=0$. Since $\tilde{K}$ is weak*-compact, we conclude that there exist $y, z$ in $\tilde{K}$ and $0<\alpha<1$ such that $x=\alpha y+(1-\alpha) z$. This contradicts the fact that $x$ is a weak*-extreme point of $K$. Thus $x \notin \overline{\mathrm{co}}\left(M_{1} \cup M_{2}\right)$. By the Hahn-Banach theorem, there exist $x^{*} \in X^{*}$ and $\delta>0$ such that $x^{*}(x)+\delta>\sup x^{*}\left(\overline{c o}\left(M_{1} \cup M_{2}\right)\right)$. Hence if $y$ is an element in the open slice $S=\left\{y: y \in K, x^{*}(y)>\sup x^{*}(K)-\delta\right\}$ then $x_{i}^{*}(y)-x_{i}^{*}(x)<\varepsilon, i=1,2$. Repeating the argument, we conclude that there is an open slice which contains $x$ and is contained in $V$. The inverse implication is obvious.

ProOF OF THEOREM. (i) $\Rightarrow$ (ii). Suppose $x$ is a denting point of $K$. Let $\left\{x_{n}\right\}$ be a sequence of $K$-valued Bochner integrable functions on $[0,1]$ such that $\lim _{n \rightarrow \infty}\left\|\int_{0}^{1} x_{n}(t) d t-x\right\|=0$. Since for every $\varepsilon>0$ there exists a simple function $y_{n}(t)$ such that $\left\|x_{n}(t)-y_{n}(t)\right\|<\varepsilon$ for all $t$, we may assume that $x_{n}(t)$ is a simple function. It follows that $\int_{0}^{1} x_{n}(t) d t=\sum_{i=1}^{k_{n}} a_{i}(n) x_{i, n}$ where $x_{i, n} \in K, a_{i}(n)>0$ and $\sum_{i=1}^{k_{n}} a_{i}(n)=1, i=1,2, \ldots, k_{n}, n=1,2, \ldots$ Suppose that

$$
\lim _{n \rightarrow \infty} \int_{0}^{1}\left\|x_{n}(t)-x\right\| d t=\lim _{n \rightarrow \infty} \sum_{i=1}^{k_{n}} a_{i}(n)\left\|x_{i, n}-x\right\| \neq 0 .
$$

By choosing a subsequence if necessary, there exists $\varepsilon>0$ such that $\sum_{i \in I_{n}} a_{i}(n)>\varepsilon$ where $I_{n}=\left\{i: 1 \leq i \leq k_{n},\left\|x_{i, n}-x\right\| \geq \varepsilon\right\}, n=1,2, \ldots$ Let $\lambda_{n}=\sum_{i \in I_{n}} a_{i}(n)$, $y_{n}=\lambda_{n}^{-1} \sum_{i \in I_{n}} a_{i}(n) x_{i, n}$ and $z_{n}=\left(1-\lambda_{n}\right)^{-1} \sum_{i \notin I_{n}} a_{i}(n) x_{i, n}$. Then $y_{n}, z_{n} \in K$ and $\sum_{i=1}^{k_{n}} a_{i}(n) x_{i, n}=\lambda_{n} y_{n}+\left(1-\lambda_{n}\right) z_{n}$. Since $x$ is a denting point of $K,\left\|y_{n}-x\right\|>$ $\delta, n=1,2, \ldots$, for some $\delta>0$. This contradicts the fact that every denting point is a strong extreme point of $K$.

It is clear that if $x$ is a very strong extreme point of $K$ then $x$ is a strong extreme point of $K$. On the other hand, it is proved in [LLT2] that if $x$ is a PC for $K$ and $x$ is an extreme point of $K$ then $x$ is a strong extreme point of $K$. It is known that $x$ is a weak*-extreme point if $x$ is a strong extreme point [KR]. Finally, we use the lemma to conclude that $x$ is a denting point of $K$.

REMARK 1. A Banach space $X$ is said to have the CPCP (convex point of continuity property) if every bounded closed convex set $K$ contains at least one PC. It is known [S] that if $X$ has CPCP then KMP is equivalent to RNP. (Recall 
a Banach space has KMP (resp. RNP) if every bounded closed convex subset of $X$ contains at least one extreme (resp. denting) point.)

REMARK 2. It can be proved directly that if $x$ is a PC for $K$ and $x$ is a strong extreme point of $K$ then $x$ is a very strong extreme point of $K$.

REMARK 3 . Let $F$ be a total subspace in $X^{*}$, and let $\tau$ be the locally convex topology on $X$ defined by $F$. By replacing $X^{*}$ by $F$ (resp. the weak topology by $\tau$ ), we may define the $\tau$-denting point (resp. $\tau$-PC point) of a bounded closed convex set $K$ in $X$. It can be proved that every $\tau$-PC, extreme point of $K$ is a strong extreme point of $K$ and that every $\tau$-PC, strong extreme point of $K$ is a $\tau$-denting point of $K$.

The authors wish to thank the referee for suggesting a direct approach in proving the theorem.

\section{REFERENCES}

[KR] Ken Kunen and Haskell Rosenthal, Martingale proofs of some geometric results in Banach space theory, Pacific J. Math. 100 (1982), 153-175.

[LL] Bor-Luh Lin and Pei-Kee Lin, Property (H) in Lebesgue-Bochner functions space, Proc. Amer. Math. Soc. 95 (1985), 581-584.

[LLT1] Bor-Luh Lin, Pei-Kee Lin and S. L. Troyanski, Some geoemtric and topological properties of the unit sphere in a Banach space, Math. Ann. 274 (1986), 613-616.

[LLT2] _ _ A characterization of denting points of a closed bounded convex set, Longhorn Notes, U. T. Functional Analysis Seminar, 1985-86, The University of Texas at Austin, pp. 99-101.

[R] Haskell Rosenthal, On the structure of nondentable closed bounded convex sets, preprint.

[S] W. Schachermayer, The Radon-Nikodým property and the Krein-Milman property are equivalent for strongly regular sets, preprint.

[T] S. Troyanski, On a property of the norm which is close to local uniform rotundity, Math. Ann. 271 (1985), 305-314.

Department of Mathematics, University of Iowa, Iowa City, IOWA 52242 (Current address of Bor-Luh Lin)

Department of Mathematics and Mechanics, University of Sofia, 5 BUl. A. IVANOV, SOFIA, BG-1126, BULGaria (Current address of S. L. Troyanski)

Current address (Pei-Kee Lin): Department of Mathematics, Memphis State University, Memphis, Tennessee 38152 